\title{
Estimation Method for Advanced Driver Assistance System and Real-Time Context-Aware
}

\author{
Byunghun Oh and Hyohaeng Lee
}

\begin{abstract}
This paper suggests an efficient estimation method for the ADAS (advanced driver assistance system) and Real-Time Context-aware. It also examines a prototype system that employs the aforementioned method. The suggested system uses the vehicle's location and driver's information to deal with accident vulnerable points. It informs drivers of appropriate distance of approach and alerts them so that drivers can efficiently recognize communication information. For the performance evaluation of the suggested system, the NASA-TLX (Task Load Index) and SUS (System Usability Scale) evaluation were performed. The results of the analysis demonstrate that this system is more efficient than existing systems.
\end{abstract}

Index Terms-Advanced driver assistance, context-aware, real-time, vehicle.

\section{INTRODUCTION}

A recent analysis of the causes of car accidents suggests that a large portion of accidents occur due to negligence, errors of judgment and delayed response by the driver. The incidence of car accidents and casualties can be organized according to the region where they occurred and according to fluctuations in annual trends. In general, the number of car ownership, and regions with higher rates of car ownership all affect the occurrence of car traffic accidents and casualties. When compared to automobile ownership and the utilization rate of automobiles, these kinds of situations result in an increased occurrence of car accidents. According to a document submitted to the 30 countries who are members of OECD (the Organization for Economic Cooperation and Development), with the exception of Mexico, the standard number of deaths per 10,000 cars was on average 1.33 in 2013 The OECD's registered car traffic accident death remains very high and is higher than the OECD's standard rate of accident deaths [1]. New technologies and diverse interfaces are being added. In addition to ordinary driving tasks, there are now additional tasks that the driver needs to handle.

In cars, the number of hands-free navigation, AV (Audio/Video) devices, information systems, entertainment, and driver assistance installation systems is increasing. However, due to an improperly-designed HMI (Human Machine Interface), the driver's safety is being seriously threatened. The diversity of information that is available

Manuscript received November 30, 2016; revised March 12, 2017. This research was supported by Basic Science Research Program through the National Research Foundation of Korea(NRF) funded by the Ministry of Education(2016R1A6A3A11930221)

Byunghun Oh is with ICT HRD Institute for New Value Creation at Sungkyunkwan University, Korea (e-mail: sincelife83@ naver.com).

Hyohaeng Lee is with PYD Corporation, Korea (e-mail: hyohaeng@skku.edu). while driving increases the driving workload. This impacts the safety and comfort of driving. When the load of information given to the driver increases, the driver's cognitive ability to process the information increases and their attention is dispersed, which then increases the driving workload. Following this, improper installation of HMI can cause errors for drivers and, in serious circumstances, accidents. In this paper, we will implement the proposed effectiveness estimation method for advanced driver assistance system and real-time context-aware. We will use NASA-TLX and SUS to evaluate it.

This paper is organized as follows: Section 2 introduces the related research. Section 3 and 4 explains the effectiveness of the estimation method for advanced driver assistance systems, as well as a real-time Context-aware description of the overall structure of each item. Section 5 presents various performance evaluations that test the effectiveness and potential applications of this research. Section 6 concludes by suggesting future areas of research.

\section{RELATED WORK}

\section{A. Subject Assessments}

First, the NASA-TLX is a widely used [2], subjective, multidimensional assessment tool that rates perceived workload in order to assess a task, system, or team's effectiveness or other aspects of performance. It was developed by the Human Performance Group at NASA's Ames Research Center over a three-year development cycle that included more than 40 laboratory simulations [3]. It has been cited in over 4,400 studies, highlighting the influence the NASA-TLX has had in human factors research. It has been used in a variety of domains, including aviation, healthcare and other complex socio-technical domains.

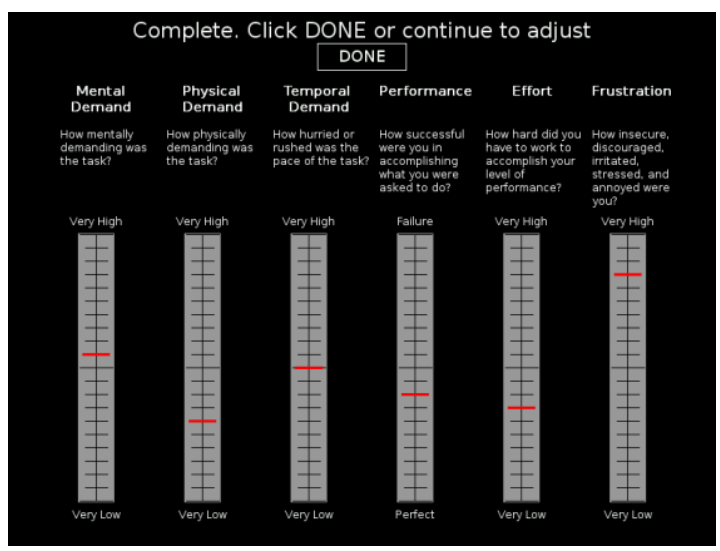

Fig. 1. The PEBL computerized version of the TLX scales 


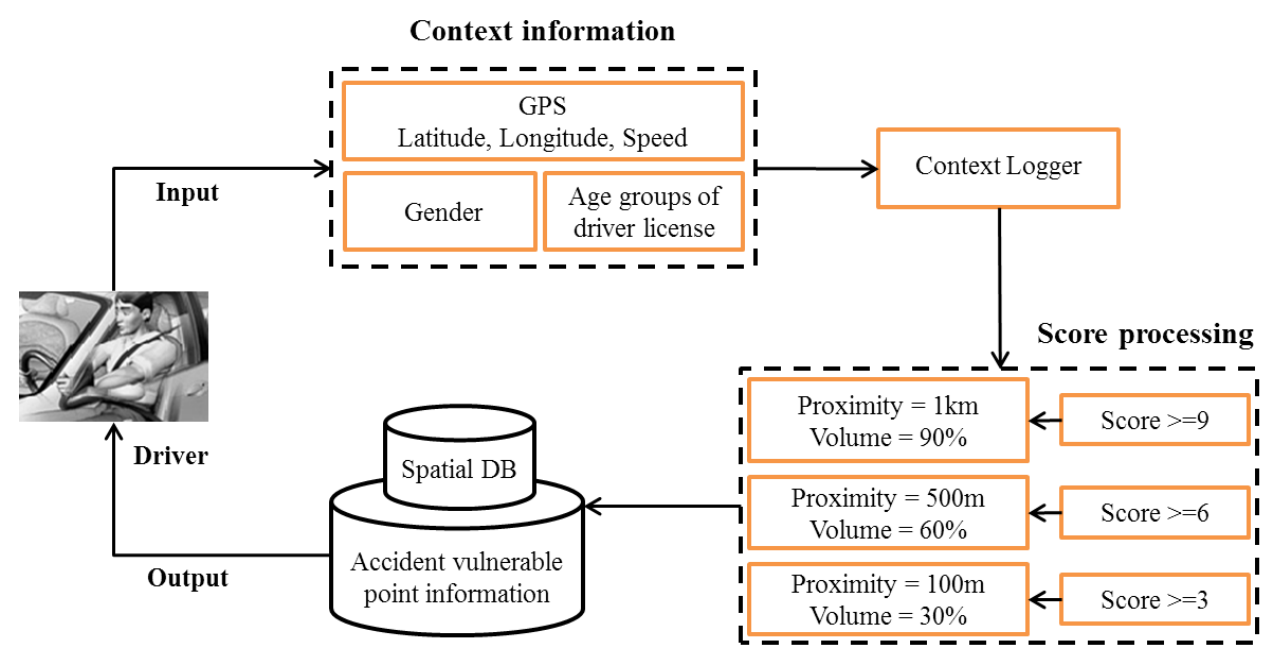

Fig. 2. System architecture of estimation method.

Second, in systems engineering, the SUS is a simple, ten-item attitude Likert scale giving a global view of subjective assessments of usability. It was developed by John Brooke [4] at Digital Equipment Corporation in the UK in 1986 as a tool to be used in usability engineering of electronic office systems.

SUS has generally been seen as providing this type of high-level subjective view of usability and is thus often used in carrying out comparisons of usability between systems. Because it yields a single score on a scale of $0-100$, it can be used to compare even systems that are outwardly dissimilar. This one-dimensional aspect of the SUS is both a benefit and a drawback, because the questionnaire is necessarily quite general. Recently, Lewis and Sauro [5] suggested a two-factor orthogonal structure, which practitioners may use to score the SUS on independent Usability and Learnability dimensions. At the same time, Borsci, Federici and Lauriola [6] by an independent analysis confirm the two factors structure of SUS also showing that those factors (Usability and Learnability) are correlated.

The SUS has been widely used in the evaluation of a range of systems. Bangor, Kortum and Miller [7] have used the scale extensively over a ten-year period and have produced normative data that allow SUS ratings to be positioned relative to other systems. They propose an extension to SUS to provide an adjective rating that correlates with a given score. Based on a review of hundreds of usability studies, Sauro and Lewis [8] proposed a curved grading scale for mean SUS scores.

\section{B. ADAS}

ADAS are designed to assist motorists while they are operating a vehicle. These systems constantly monitor a number of parameters and when thresholds are exceeded, drivers are informed [9].

The driver of the car constantly reads the incoming visual information as he correctly judges the situation, while completing his feedback to the car through repeated manipulation, although he is not using large muscles of his hands or feet, instead he is using his brain's activity to process his information [10].

In vehicle-pedestrian collisions, it has been revealed that most times, the pedestrian collided with the vehicle head on. According to the results of an analysis of pedestrian injuries in collision by the U.S. NHTSA (National Highway Traffic Safety Administration), $71.1 \%$ of pedestrian had a head on collision with the vehicle. Also, if the pedestrian died, only $80 \%$ of pedestrians had a head on collision with the vehicle [11]. We can say that the most basic feature of navigation is to guide from the current location to the destined location. Also its features can be divided into identifying the current location and displaying the current location, searching the destination from maps, searching routes to destination, and identifying roads and guiding to routes [12].

In order to provide these kinds of features, the elements of these features have location information technology, route navigation and guidance technology map-matching technology, user interface technologies such as speech recognition and synthesis, information management, and real-time traffic information, etc. includes wireless communication technology [13]. These kinds of services have a limitation of not being able to provide situational information to drivers.

Therefore, it increases the user's cognitive load, and the driver safety is threatened. In addition, the existing navigation demands manual setting not considering the situation of the driver in delivering warning and information and provides information to the driver on the basis of the set information [14], [15]. We would like to propose and implement the estimation method for advanced driver assistance system as one of the methods.

\section{SYSTEM ARCHITECTURE}

Fig. 2 showed system architecture of estimation method, the proposed method is performed to the following steps. It obtains GPS (Global Positioning System) information (such as latitude and longitude), gender, and the age of the license holder before calculating the current speed of the car. It then puts the situation log together.

It displays information by using the accident weak point and space information. On the basis of the score information and location information, it creates a situation $\log$ and, following the score and volume, outputs the warning alarm 
near the accident weak point.

By applying the real-time score, the method suggested uses the ratio of drivers with license according to gender, the number of accidents of drivers with license according to gender, the number of drivers with license according to gender, and location information (such as latitude, longitude and speed) to provide an alarm and volume that are proper for the driver's situation. A real-time calculation is conducted using latitude and longitude, in addition to other types of GPS information, to get the nearest distance between weak points for accidents and the driver [16].

\section{CONTEXT-AwARE PROCESS}

The situation information data is organized according to gender, age of license holder, latitude, longitude, and speed. In this paper, the TAAS (Traffic Accident Analysis System) provided by Korea Road Traffic Authority was used, and the TAAS used gender, age of license holder, and accident records [17].

TABLE I: TRAFFIC ACCIDENT ANALYSIS STATISTICS

\begin{tabular}{ccccc}
\hline \hline Gender & $\begin{array}{c}\text { Driver's } \\
\text { license }\end{array}$ & $\begin{array}{c}\text { Driver's license } \\
\text { accident }\end{array}$ & Rate & Score \\
\hline Male & $16,074,165$ & 182,777 & 0.001137085 & 2 \\
Female & $10,328,199$ & 37,127 & 0.003594721 & 1 \\
Sum & $26,402,364$ & 219,904 & & \\
\hline \hline
\end{tabular}

Table I shows the number of all drivers with license following the gender with TAAS data as the grounds, and the number of accidents of drivers with license following the gender is based on the accident data of 2015. The rate was calculated on the basis of ground data and the score was calculated.

TABLE II: TRAFFIC ACCIDENT ANALYSIS STATISTICS

\begin{tabular}{ccccc}
\hline \hline Ages & $\begin{array}{c}\text { Driver's } \\
\text { license }\end{array}$ & $\begin{array}{c}\text { Driver's } \\
\text { license } \\
\text { accident }\end{array}$ & Rate & Score \\
\hline 16-19 years & 274,715 & 7,815 & 0.028447663 & 6 \\
20-29 years & $4,522,216$ & 33,180 & 0.007337110 & 2 \\
30-39 years & $7,082,677$ & 44,729 & 0.006315267 & 1 \\
40-49 years & $7,159,013$ & 60,042 & 0.008386910 & 3 \\
$50-59$ years & $4,883,426$ & 48,988 & 0.010031481 & 5 \\
60 years of age & $2,480,317$ & 25,150 & 0.010139832 & 4 \\
or older & & 219,904 & & \\
Sum & $26,402,364$ & & & \\
\hline \hline
\end{tabular}

Table II divides the age of all the drivers with a license into 6 stages with TAAS data as the grounds. The rate was calculated using the number of all drivers with a license in each age range and the number of accidents of drivers with licenses in each age range in 2015. The rate following the gender of drivers with licenses and the number of accidents of drivers with a license is the information that is needed for situation recognition. The rate of the number of drivers with a license following age and the number of accidents of drivers with a license are used. The score is calculated together with the speed obtained from the GPS.

The standard calculation of speed is as follows:

- 1 when $0 \mathrm{~km} \sim 30 \mathrm{~km}$

- 2 when $31 \mathrm{~km} \sim 60 \mathrm{~km}$

- 3 when $61 \mathrm{~km} \sim$ over speed

The neighboring warning and volume that are suitable to the situation are outputted in real time by adding the scores of Table I and Table II.

\section{EXPERIMENT AND RESULTS}

A field test was conducted with 6 men and 6 women. For the performance evaluation of the proposed method, the subjects were composed of driver's license holders from each age group. The NASA-TLX [18] for the driving work load evaluation and the SUS [19] survey for the system effectiveness evaluation were applied in order to evaluate the driving work load and the effectiveness after the field test. In the field test, cars were travelling a distance of $5 \mathrm{~km}$ and the travel speed between $30 \mathrm{~km}$ and $80 \mathrm{~km}$.

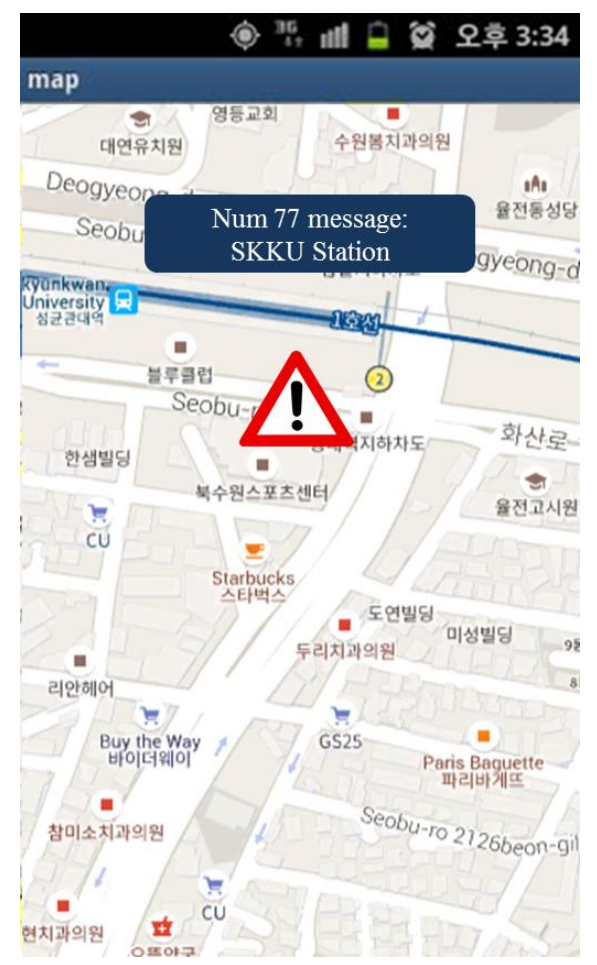

Fig. 3. Example of proposed results.

Figure 3 displays the gender and age of the driver and calculates the real-time score when approaching the point weak for accidents following the situation of the driver. Then the system outputs the warning image and alarm at a proper point in time. The system also outputs a warning image and alarm when the real-time score changes.

The results of the survey are shown in Fig. 4 and Fig. 5. Figure 1 shows the SUS evaluation to be $83 \%$ and the average for NASA-TLX to be $33 \%$. There is a correlation in the aspect of mutual evaluation. In conclusion, the system under evaluation in this paper is effective for the driver, with an SUS 
average of $83 \%$. It is also known that the driving workload is low, with an NASA-TLX average of 33\%. Furthermore, it should be known that it is valid to grasp the appropriateness of the system in the aspect of the driver.

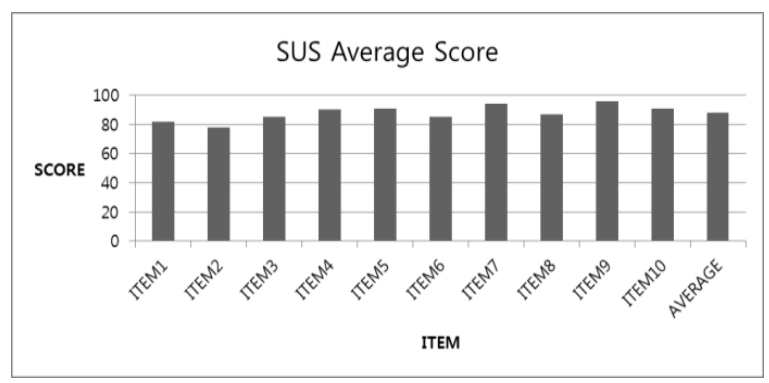

Fig. 4. SUS average score.

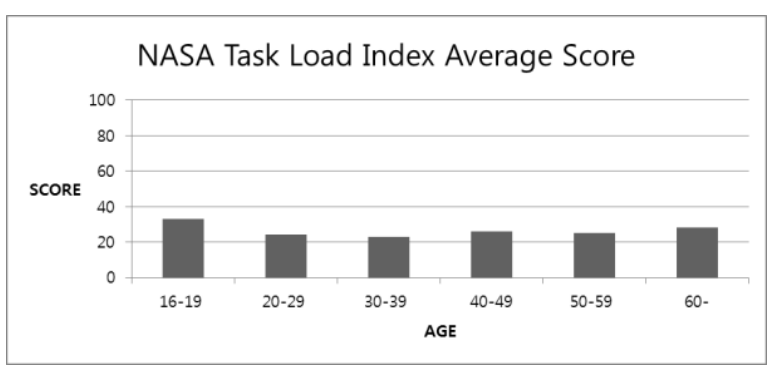

Fig. 5. NASA-TLX average score.

\section{SUMMARIES}

This paper proposes and implements the effectiveness estimation method for an advanced driver assistance system and real-time context-aware. The result of NASA-TLX and SUS has been found to be average $33 \%$ and $83 \%$. The proposed method will allow the driver to drive efficiently and easily handle dangerous situations.

This paper proposed an intelligent interface system that provides the proper information to the driver at a suitable time and place and can control the intensity for the driver as the occasion demands. When realized, this system will be the basis for an intelligent situation recognition system that selects the modality intensity following the driver. In addition, by providing a fusion of variety of vehicle's real-time information and the driver information, we can be improved cognitive ability.

Due to the complicated nature of human beings, longitudinal driving behavior is affected by many factors such as driver physical and mental characteristics, vehicle types, and road environment (including road geometry, weather conditions, traffic information, and real-time traffic flow). To further enhance the driver acceptance of $\mathrm{ADAS}$, further research into different weather conditions under different road and traffic conditions will be conducted in the future.
[2] L. Colligan, H. Potts and CT. Finn, "Cognitive workload changes for nurses transitioning from a legacy system with paper documentation to a commercial electronic health record," International Journal of Medical Informatics, no. 7, pp. 469-476, July 2015.

[3] NASA, "Nasa Task Load Index (TLX) v. 1.0 Manual", 1986.

[4] J. Brooke, "SUS: A "quick and dirty" usability scale," In P. W. Jordan, B. Thomas, B. A. Weerdmeester, \& A. L. McClelland, 1996.

[5] J.R., Lewis, and J. Sauro, " The factor structure of the system usability scale," International Conference (HCII 2009), San Diego CA, USA, 2009.

[6] S. Borsci, S. Federici, and M. Lauriola, "On the dimensionality of the system usability scale: A test of alternative measurement models," Cognitive Processing, no. 3, pp. 193-197, 2009.

[7] T. Philip and T. M. James, "An empirical evaluation of the system usability scale," International Journal of Human-Computer Interaction, no. 6, pp. 574-594, 2008.

[8] J. Sauro, and J. R. Lewis, "Quantifying the user experience: Practical statistics for user research," Morgan Kaufmann, Waltham MA, USA, 2012.

[9] N. Merat, J. D, Lee, "Preface to the special section on suman factors and automation in vehicles: designing highly automated vehicles with the driver in mind," Hum. Factors J. Hum. Factors Ergon. Soc, no.5, pp. 681-686, 2012.

[10] K. S. Park and T. Ohkubo, "A study on visual characteristics of train drivers for the differences of display layout," The Japanese Journal of Ergonomics, no. 1, pp.31-38, 2010.

[11] J. R. Crandall, K. S. Bhalla, and N. J. Madeley, "Designing road vehicles for pedestrian protection," Brit. Med., no. 5, pp. 1145-1148, 2002.

[12] U. Yoshinobu. The 2nd Car Navigation. [Online]. Available: http://www.its-lectures.ae.keio.ac.jp/2001/2001_eb_2.htm

[13] Y. Zhao, Vehicle Location and Navigation Systems, Boston, MA, Artech House, Inc, 1997.

[14] Inavi. [Online]. Available: https://www.inavi.com/_Menu/Customer/ prod_manual.asp

[15] Mio. [Online]. Available: https://www.mio.com.cn/

[16] Hexa software development. Geographical distance calculations. [Online]. Available: http://www.zipcodeworld.com

[17] TAAS. [Online]. Available: http://taas.koroad.or.kr/indexMain.jsp

[18] NASA TLX: task load index. [Online]. Available: http://human-factors.arc.nasa.gov/groups/TLX/

[19] J. Brooke, "SUS: A quick and dirty usability scale," Usability Evaluation in Industry, London: Taylor and Francis, pp. 189-194, 1996.

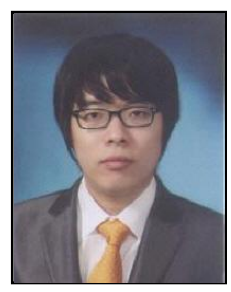

ByungHun Oh is a post-doc (research fellow) in ICT HRD Institute for New value Creation at Sungkyunkwan University. His received the B. S. degrees in electronic engineering from the Eulji University, in 2011 and his M.S. and Ph.D. from the Department of Electrical and Computer Engineering at Sungkyunkwan University in 2013 and 2016. His current research focuses on digital image processing and pattern recognition.

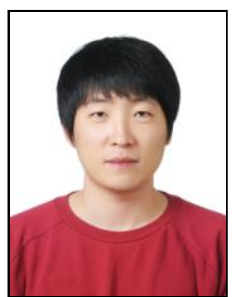

HyoHaeng Lee is the CEO at PYD Corporation. His received the $\mathrm{B}$. S. degrees in electronic engineering from the Hongik University, in 2006 and his M.S. and $\mathrm{Ph} . \mathrm{D}$. from the Department of Electrical and Computer Engineering at Sungkyunkwan University in 2009 and 2015. His current research focuses on artificial intelligence and human computer interaction.

\section{REFERENCES}

[1] I. Dadger and T. Norstrom, "Short-term and long-term effects of GDP on traffic deaths in 18 OECD countries, 1960-2011," J Epidemiol Comnnunity Health, Published Online First: 16, August 2016. 